\title{
Evaluation of Rice Genotypes for Seed Morphometric Characters by Using Seed Image Analyser
}

\author{
Elizabeth $^{1^{*}}$, N.M. Shakuntala ${ }^{2}$, Basave Gowda ${ }^{3}$, K. Mahantashivayogayya ${ }^{4}$, \\ J.R. Dewan ${ }^{5}$ and I.M. Sangeeta ${ }^{6}$
}

${ }^{1}$ Department of Seed Science and Technology, AC Raichur, UAS Raichur, Karnataka, India

${ }^{2}$ Department of Seed Science and Technology, AC Raichur, Karnataka, India

${ }^{3}$ Special Officer (Seeds) UAS, Raichur, Karnataka, India

${ }^{4}$ ARS Gangavathi, Department of Genetics and Plant Breeding, AC Raichur, UAS, Raichur, Karnataka, India

${ }^{5}$ Department of Genetics and Plant Breeding, ${ }^{6}$ Department of Seed Science and Technology, AC, UAS, Raichur, Karnataka, India

*Corresponding author

\section{A B S T R A C T}

\section{Keywords}

Rice (Oryza sativa

L.), Global grain,

Seed shape

Article Info

Accepted:

24 August 2018

Available Online:

10 December 2018
An image analysis technique is well suited for the collection of data concerning the shape and size of the seeds. Such morphological data is often used for establishing the distinctness of new varieties. Seed morphometric characters like seed length, breadth, length to breadth ratio (seed shape) and thousand seed weight were determined for hundred rice genotypes by using Biovis image analyser. As seed size is a widely accepted measure of seed quality by different scientists, study was conducted to determine whether seed size really affects seedling establishment and to its vigour in rice genotypes. Digital image analysis has proven to be a viable approach for the quantitative characterization of cultivars. It helps in distinguishing the cultivars, representing the separation of short, medium and long, as well as brown and white rice varieties using a combination of the descriptors including average length, breadth, shape factor, and compactness.

\section{Introduction}

Rice (Oryza sativa L.) is a "Global Grain" cultivated widely across the world and feeds millions of mankind. It is the staple food for more than half of the human population and in Asia alone more than 2 billion people depend on rice and its products for their food intake. Seed morphometric characters viz., seed size, seed width, seed length to breadth ratio (seed shape) and thousand seed weight were determined, the genotype Gangavathi emergency was found to be numerically highest and IET-220551 genotype was found to be numerically lowest in all the seed characters and were grouped into different categories. It was noticed that seed size is related with seed vigour, larger seeds tend to produce more vigorous seedlings. The seed size indicated the amount of reserve food 
supply for seedlings. Small and shrivelled seeds do not contain as much food to give the plant a vigorous start that of bold and plumby seeds. Seed size is the main factor that affects seeding rates. Seed morphometric characters play an important role in characterization and grouping of rice genotypes.

Accurate identification of genotypes or varieties for assessment of genetic variability is very useful from initial parent selection to the final utilization of cultivars in production schemes. Diversity analysis is an essential process for proper identification of the genetic relatedness of the available genetic resources. It is also required for effective choice of parents for subsequent crossing and selection of the progenies. Morphological or phenotypic descriptors have traditionally been used to distinguish one accession from the other but they are subjected to environmental influences.

\section{Materials and Methods}

Hundred rice genotypes viz., IET-23304, IET26286, IET-24796, IET-22066, IET-25576, IET-25546, IET-26282, IET-26234, IET22067, IET-25577, IET-22051, IET-24053, IET-24798, IET-24799, IET-25597, IET25677, IET-25548, IET-25551, IET-25598, IET- 25583, IET-24796, IET-25557, IET23356, IET-23748, IET-26217, IET-26218, IET-26219, IET-26222, IET-26225, IET25546, IET-26230, IET-26231, IET-26232, IET-26233, IET-26223, IET-26280, IET26281, IET-26283, IET-26294, IET-26295, IET-26296, IET-23304, IET-26240, IET23355, IET-24705, IET-25605, IET-24903, IET-24911, IET-25557, IET-25559, IET25580, IET-24708, IET-25584, IET-23996, IET-26220, IET-26221, IET-26224, IET26226, IET-26227, IET-26228, IET-26290, IET-26291, IET-26292, IET-26293, IET23324, MSB-43-1-2, 23959, IR-72875-53, IET-26238, IET-26239, IET-26241, IET-
26242, IET-26243, SMW-09-32, GNV-10-89, IET-26235, IET-26236, IET-26277, IET26289, DRR-Dhan-44, Gangavathi emergency, GNV-11-09, IABT-17, RP-Bio26, IET-24767, IET-26237, PR-124, GNV-1496-1, GNV-1415, KRGL-20, 26237, GNV1405 and Biovis seed image analyser which is used as an image processing programme, less time consuming system, were used in this study and were maintained at Department of Seed Science and Technology, College of Agriculture, University of Agricultural Sciences, Raichur. A range of seed traits were measured using the procedures described below.

\section{Morphological characters}

Seed morphological characters viz., length $(\mathrm{mm})$, breadth $(\mathrm{mm})$ and length to breadth ratio was measured by taking ten seeds with ten replications using Biovis image analyser and were grouped into different categories. For seed length they were grouped into short $(<7.5 \mathrm{~mm})$, medium $(7.5-9 \mathrm{~mm})$, long (9$10 \mathrm{~mm})$ and very long $(>10 \mathrm{~mm})$, for seed breadth the genotypes were classified into narrow (1.9-2.2mm), medium (2.2-2.8mm), broad $(>2.88 \mathrm{~mm})$ and elongated $(>3 \mathrm{~mm})$ groups and for seed length to breadth ratio (seed shape) they were grouped into spherical $(<2)$, semi spherical (2-2.4) and semi long (2.4-3) categories. Seed colour was observed under daylight and were grouped into different groups viz., straw, golden and yellow colour.

\section{Results and Discussion}

Rice genotypes used for the study varied significantly for seed length, seed breadth and seed length to breadth ratio (seed shape). The highest seed length, breadth and length to breadth ratio was observed in Gangavathi emergency $(11.29 \mathrm{~mm}),(3.6 \mathrm{~mm})$ and $(3.65)$ lowest was found in IET-255051 $(7.01 \mathrm{~mm})$, $(1.90 \mathrm{~mm})$ and $(2.32)$ respectively (Table 1$)$. 
Table.1 Grouping of rice genotypes based on seed morphometric characters

\begin{tabular}{|c|c|c|c|c|c|c|c|c|c|c|}
\hline $\begin{array}{l}\text { SI. } \\
\text { no. }\end{array}$ & Genotypes & $\begin{array}{c}\text { Seed } \\
\text { length } \\
(\mathbf{m m})\end{array}$ & Category & $\begin{array}{c}\text { Seed } \\
\text { breadth } \\
(\mathrm{mm})\end{array}$ & Category & $\begin{array}{c}\text { Seed } \\
\text { length/ } \\
\text { breadth } \\
\text { ratio }\end{array}$ & Category & Seed colour & $\begin{array}{l}1000 \text { seed } \\
\text { weight } \\
\text { (g) }\end{array}$ & Category \\
\hline 1 & IET-24053 & 8.24 & Medium & 2.38 & Medium & 3.53 & Elongated & Yellow colour & 14.72 & Light \\
\hline 2 & IET-23304 & 7.47 & Short & 2.17 & Narrow & 3.55 & Elongated & Straw colour & 14.88 & Light \\
\hline 3 & IET-24705 & 9.06 & Long & 3.16 & Broad & 2.91 & Semi long & Straw colour & 17.52 & Light \\
\hline 4 & IET-26286 & 7.29 & Short & 2.09 & Narrow & 3.56 & Elongated & Yellow colour & 14.18 & Light \\
\hline 5 & IET-24798 & 8.99 & Medium & 2.57 & Medium & 3.54 & Elongated & Yellow colour & 18.71 & Medium \\
\hline 6 & IET-24796 & 7.46 & Short & 2.16 & Narrow & 3.21 & Elongated & Golden colour & 17.13 & Light \\
\hline 7 & IET-24799 & 8.32 & Medium & 3.57 & Broad & 2.32 & Semi spherical & Straw colour & 20.03 & Medium \\
\hline 8 & IET-22066 & 7.18 & Short & 2.19 & Narrow & 3.26 & Elongated & Yellow colour & 14.95 & Light \\
\hline 9 & IET-25597 & 8.42 & Medium & 2.23 & Medium & 3.75 & Elongated & Straw colour & 21.13 & Medium \\
\hline 10 & IET-25600 & 8.83 & Medium & 2.74 & Medium & 3.17 & Elongated & Straw colour & 21.08 & Medium \\
\hline 11 & IET-25605 & 9.41 & Long & 2.80 & Medium & 3.39 & Elongated & Golden colour & 14.96 & Light \\
\hline 12 & IET-25548 & 8.77 & Medium & 2.64 & Medium & 3.35 & Elongated & Golden colour & 14.15 & Light \\
\hline 13 & IET-25576 & 7.01 & Short & 2.12 & Narrow & 2.92 & Semi long & Straw colour & 17.15 & Light \\
\hline 14 & IET-24903 & 9.52 & Long & 3.38 & Broad & 2.85 & Semi long & Straw colour & 19.19 & Medium \\
\hline 15 & IET-24911 & 9.12 & Long & 3.07 & Broad & 2.74 & Semi long & Yellow colour & 15.55 & Light \\
\hline 16 & IET-25551 & 8.96 & Medium & 2.29 & Medium & 3.99 & Elongated & Straw colour & 16.26 & Light \\
\hline 17 & IET-25598 & 8.59 & Medium & 2.42 & Medium & 3.61 & Elongated & Yellow colour & 15.18 & Light \\
\hline 18 & IET-24902 & 9.18 & Long & 3.37 & Broad & 2.78 & Semi long & Straw colour & 16.27 & Light \\
\hline $\begin{array}{c}\text { Sl } \\
\text { no. }\end{array}$ & Genotypes & $\begin{array}{c}\text { Seed } \\
\text { length } \\
(\mathbf{m m})\end{array}$ & Category & $\begin{array}{c}\text { Seed } \\
\text { breadth } \\
(\mathbf{m m})\end{array}$ & Category & $\begin{array}{c}\text { Seed } \\
\text { length/ } \\
\text { breadth } \\
\text { ratio }\end{array}$ & Category & Seed colour & $\begin{array}{l}1000 \text { seed } \\
\text { weight } \\
\text { (g) }\end{array}$ & Category \\
\hline 19 & IET-25582 & 8.94 & Medium & 3.13 & Broad & 3.41 & Elongated & Yellow colour & 17.75 & Light \\
\hline 20 & IET-25557 & 9.24 & Long & 3.26 & Broad & 2.89 & Semi long & Yellow colour & 23.51 & Heavy \\
\hline 21 & IET-25559 & 9.12 & Long & 3.35 & Broad & 2.77 & Semi long & Yellow colour & 17.12 & Light \\
\hline 22 & IET-24796 & 7.53 & Medium & 2.23 & Medium & 3.88 & Elongated & Straw colour & 14.09 & Light \\
\hline 23 & IET-25552 & 8.01 & Medium & 2.49 & Medium & 3.65 & Elongated & Straw colour & 19.55 & Medium \\
\hline
\end{tabular}




\begin{tabular}{|c|c|c|c|c|c|c|c|c|c|c|}
\hline 24 & IET-23356 & 8.66 & Medium & 2.28 & Medium & 3.88 & Elongated & Straw colour & 23.46 & Heavy \\
\hline 25 & IET-23748 & 8.52 & Medium & 2.67 & Medium & 3.26 & Elongated & Yellow colour & 16.17 & Light \\
\hline 26 & IET-25580 & 9.46 & Long & 3.42 & Broad & 2.81 & Semi long & Yellow colour & 16.13 & Light \\
\hline 27 & IET-24708 & 9.18 & Long & 3.05 & Broad & 2.86 & Semi long & Straw colour & 19.87 & Medium \\
\hline 28 & IET-25584 & 9.55 & Long & 3.31 & Broad & 2.95 & Semi long & Straw colour & 20.28 & Medium \\
\hline 29 & IET-25546 & 7.11 & Short & 2.20 & Narrow & 2.99 & Semi long & Yellow colour & 20.96 & Medium \\
\hline 30 & IET-23996 & 9.17 & Long & 3.62 & Broad & 2.57 & Semi long & Golden colour & 20.87 & Medium \\
\hline 31 & IET-26217 & 8.94 & Medium & 2.37 & Medium & 3.81 & Elongated & Straw colour & 17.33 & Light \\
\hline 32 & IET-26218 & 8.85 & Medium & 2.71 & Medium & 3.31 & Elongated & Golden colour & 17.11 & Light \\
\hline 33 & IET-26219 & 8.16 & Medium & 2.59 & Medium & 3.42 & Elongated & Straw colour & 13.15 & Light \\
\hline 34 & IET-26220 & 9.16 & Long & 3.56 & Broad & 2.61 & Semi long & Straw colour & 12.77 & Light \\
\hline 35 & IET-26221 & 9.13 & Long & 3.46 & Broad & 2.68 & Semi long & Golden colour & 21.18 & Medium \\
\hline 36 & IET-26222 & 8.45 & Medium & 2.81 & Broad & 3.06 & Elongated & Golden colour & 20.19 & Medium \\
\hline 37 & IET-25546 & 7.69 & Medium & 2.29 & Medium & 3.23 & Elongated & Straw colour & 14.20 & Light \\
\hline 38 & IET-26224 & 9.16 & Long & 3.24 & Broad & 2.87 & Semi long & Straw colour & 19.28 & Medium \\
\hline 39 & IET-26225 & 8.7 & Medium & 2.54 & Medium & 3.45 & Elongated & Yellow colour & 17.06 & Light \\
\hline $\begin{array}{r}\text { SI } \\
\text { no. }\end{array}$ & Genotypes & $\begin{array}{c}\text { Seed } \\
\text { length } \\
(\mathbf{m m})\end{array}$ & Category & $\begin{array}{c}\text { Seed } \\
\text { breadth } \\
(\mathrm{mm})\end{array}$ & Category & $\begin{array}{c}\text { Seed } \\
\text { length/ } \\
\text { breadth } \\
\text { ratio }\end{array}$ & Category & Seed colour & $\begin{array}{l}1000 \text { seed } \\
\text { weight } \\
\text { (g) }\end{array}$ & Category \\
\hline 40 & IET-26226 & 9.14 & Long & 3.25 & Broad & 2.86 & Semi long & Yellow colour & 34.05 & Heavy \\
\hline 41 & IET-26227 & 9.24 & Long & 3.22 & Broad & 2.92 & Semi long & Yellow colour & 18.77 & Medium \\
\hline 42 & IET-26228 & 9.85 & Long & 3.08 & Broad & 3.24 & Elongated & Golden colour & 18.16 & Medium \\
\hline 43 & GNV-10-89 & 10.96 & Very long & 3.13 & Broad & 3.53 & Elongated & Straw colou & 26.16 & Heavy \\
\hline 44 & IET-26229 & 9.94 & Long & 3.43 & Broad & 3.04 & Elongated & Straw colour & 17.45 & Light \\
\hline .45 & IET-26230 & 8.72 & Medium & 2.20 & Narrow & 3.94 & Elongated & Straw colour & 19.25 & Medium \\
\hline 46 & IET-26231 & 8.95 & Medium & 2.58 & Medium & 3.52 & Elongated & Golden colour & 18.64 & Medium \\
\hline 47 & IET-26232 & 8.29 & Medium & 2.64 & Medium & 3.41 & Elongated & Golden colour & 21.09 & Medium \\
\hline 48 & IET-26233 & 8.88 & Medium & 2.24 & Medium & 4.04 & Elongated & Yellow colour & 20.12 & Medium \\
\hline 49 & IET-26235 & 10.11 & Very long & 3.62 & Broad & 2.79 & Semi long & Yellow colour & 19.49 & Medium \\
\hline 50 & IET-26236 & 10.15 & Very long & 3.11 & Broad & 3.28 & Elongated & Straw colour & 17.17 & Light \\
\hline 51 & IET-26277 & 10.07 & Very long & 2.92 & Broad & 3.48 & Elongated & Golden colour & 24.25 & Heavy \\
\hline 52 & IET-26278 & 9.76 & Long & 2.85 & Broad & 3.47 & Elongated & Straw colour & 23.24 & Heavy \\
\hline
\end{tabular}




\begin{tabular}{|c|c|c|c|c|c|c|c|c|c|c|}
\hline 53 & IET-26279 & 9.54 & Long & 3.13 & Broad & 3.09 & Elongated & Yellow colour & 24.12 & Heavy \\
\hline 54 & IET-26280 & 8.07 & Medium & 2.26 & Medium & 3.97 & Elongated & Straw colour & 17.07 & Light \\
\hline 55 & IET-26223 & 8.14 & Medium & 2.84 & Broad & 2.92 & Semi long & Yellow colour & 14.16 & Light \\
\hline 56 & IET-26281 & 8.38 & Medium & 2.34 & Medium & 3.61 & Elongated & Yellow colour & 17.94 & Light \\
\hline 57 & IET-26282 & 7.22 & Short & 2.09 & Narrow & 2.61 & Semi long & Golden colour & 14.09 & Light \\
\hline 58 & IET-26283 & 8.91 & Medium & 2.52 & Medium & 3.55 & Elongated & Golden colour & 19.49 & Medium \\
\hline 59 & IET-26284 & 9.14 & Long & 1.97 & Narrow & 2.80 & Semi long & Straw colour & 23.46 & Heavy \\
\hline 60 & IET-26285 & 9.01 & Long & 1.95 & Narrow & 2.83 & Semi long & Yellow colour & 19.57 & Medium \\
\hline $\begin{array}{c}\text { SI } \\
\text { no. }\end{array}$ & Genotypes & $\begin{array}{c}\text { Seed } \\
\text { length } \\
(\mathbf{m m})\end{array}$ & Category & $\begin{array}{c}\text { Seed } \\
\text { breadth } \\
(\mathrm{mm})\end{array}$ & Category & $\begin{array}{c}\text { Seed } \\
\text { length/ } \\
\text { breadth } \\
\text { ratio }\end{array}$ & Category & Seed colour & $\begin{array}{c}1000 \text { seed } \\
\text { weight } \\
\text { (g) }\end{array}$ & Category \\
\hline 61 & IET-26287 & 9.16 & Long & 2.11 & Narrow & 2.82 & Semi long & Straw colour & 18.27 & Medium \\
\hline 62 & IET-26288 & 9.18 & Long & 1.95 & Narrow & 2.81 & Semi long & Yellow colour & 17.78 & Medium \\
\hline 63 & IET-26289 & 10.16 & Very long & 2.05 & Narrow & 3.22 & Elongated & Yellow colour & 17.17 & Medium \\
\hline 64 & DRR- Dhan-44 & 11.13 & Very long & 3.54 & Broad & 3.38 & Elongated & Straw colour & 29.16 & Heavy \\
\hline 65 & Gangavathi emergency & 11.29 & Very long & 3.66 & Broad & 3.65 & Elongated & Golden colour & 32.14 & Heavy \\
\hline 66 & GNV-11-09 & 10.08 & Very long & 3.63 & Broad & 3.03 & Elongated & Golden colour & 26.28 & Heavy \\
\hline 67 & IET-26290 & 9.99 & Long & 3.27 & Broad & 3.08 & Elongated & Straw colour & 16.36 & Light \\
\hline 68 & IET-26291 & 9.31 & Long & 3.49 & Broad & 2.79 & Semi long & Straw colour & 16.31 & Light \\
\hline 69 & IET-26292 & 9.39 & Long & 3.23 & Broad & 2.93 & Semi long & Straw colour & 17.94 & Light \\
\hline 70 & IET-26293 & 9.19 & Long & 3.53 & Broad & 2.64 & Semi long & Golden colour & 16.27 & Light \\
\hline 71 & IET-26294 & 8.58 & Medium & 2.06 & Narrow & 3.91 & Elongated & Golden colour & 21.12 & Medium \\
\hline 72 & IET-26295 & 8.72 & Medium & 2.93 & Broad & 3.04 & Elongated & Yellow colour & 16.28 & Light \\
\hline 73 & IET-26234 & 7.33 & Short & 2.14 & Narrow & 3.22 & Elongated & Golden colour & 14.08 & Light \\
\hline 74 & IET-22066 & 7.21 & Short & 2.02 & Narrow & 2.79 & Semi long & Straw colour & 14.18 & Light \\
\hline 75 & IET-26296 & 8.18 & Medium & 2.24 & Medium & 3.73 & Elongated & Straw colour & 17.14 & Light \\
\hline 76 & IET-23304 & 8.69 & Medium & 2.44 & Medium & 3.61 & Elongated & Straw colour & 16.19 & Light \\
\hline 77 & IET-25577 & 7.48 & Short & 2.57 & Medium & 3.74 & Elongated & Straw colour & 14.19 & Light \\
\hline 78 & IET-23324 & 9.19 & Long & 3.17 & Broad & 2.94 & Semi long & Yellow colour & 19.98 & Medium \\
\hline 79 & MSB-43-1-2 & 9.97 & Long & 3.39 & Broad & 2.99 & Semi long & Straw colour & 20.17 & Medium \\
\hline 80 & IET-23959 & 9.28 & Long & 3.61 & Broad & 2.61 & Semi long & Yellow colour & 20.91 & Medium \\
\hline SI & Genotypes & Seed & Category & Seed & Category & Seed & Category & Seed colour & 1000 & Category \\
\hline
\end{tabular}




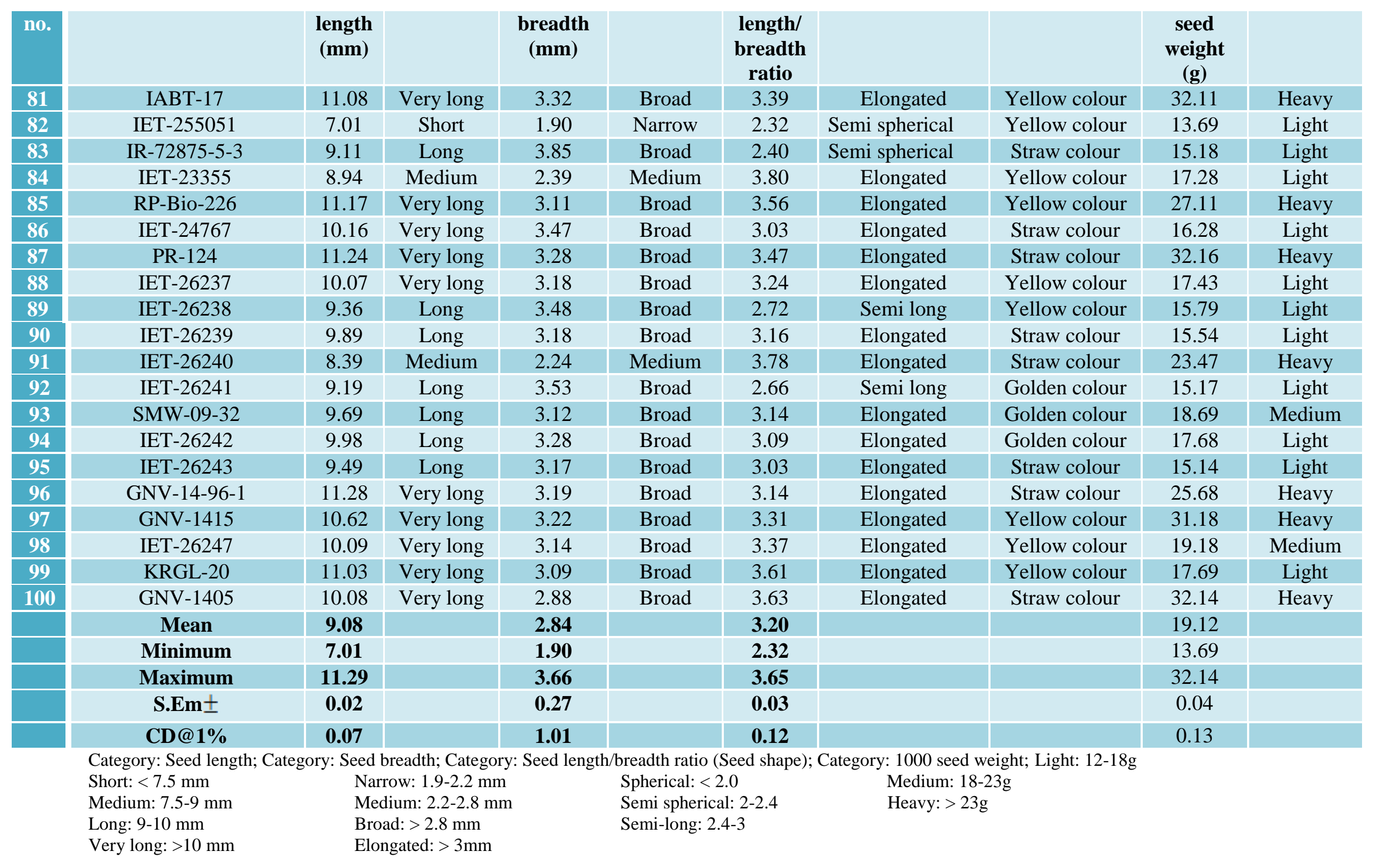


Based on the mean of seed length, breadth and shape the genotypes were grouped into different categories.

Seed morphological studies can be made automatically by using Image Analysis System, i.e., by Biovis Seed Image Analyser. Seed morphological characters viz., size, length, breadth, length to breath ratio and colour were used for genotype characterization.

A digital image analysis provides an alternative to the manual classification of biological seed by integrating an image acquisition device and a computer. Similar works were carried out by Mattana et al., (2008), Firathgil et al., (2010), Chavan (2010), Medina et al., (2010), Bacchetta et al., (2011) and Grillo et al., (2011).

Genetic analysis of these two quantitative traits have been reported by many scientists and most have shown that grain shape of rice is quantitatively inherited Zhang et al., (2007) showed that rice grain shape is controlled by triploid endosperm gene, cytoplasmic gene and genotype by environment interaction.

Based on the colour of the seed, the genotypes were classified into three groups golden colour, straw colour and yellow colour. However, the seed colour is also influenced by environmental conditions during ripening, besides the genetic effect (Pascual et al., 1993). Thus, it is important to note that such comparisons are valid only, if the crop is not adversely affected by rains, moulds or any other biotic and abiotic stresses and the crop is grown under similar agro-climatic conditions. Seed colour is considered to be a heritable character and has been used by several scientists to distinguish crop genotypes (Chakrabarthy and Agarwal, 1990; Agarwal and Pawar, 1990), particularly in rice (Nethra, 2003 and Rimpi, 2008).
The genotype Gangavathi emergency possess highest while IET-255051 possess lowest seed length, seed breadth, seed length to breadth ratio, other different rice genotypes. Seeds having more seed weight and seed size have higher seedling vigour which are preferred for better crop establishment. Seed size will be more influential parameter for processing and fixing the seed rate. Hence each variety will be having varied seed rates. The study helps to know the vigour, seed rate, processing designing and fineness for consumption. It is useful for seed industry as well as commercial food industry. An image analysis technique is well suited for the collection of data concerning the shape and size of objects and this applies to plant organs as well as seeds. Such morphological data is often used for establishing the distinctness of new varieties in statutory plant breeding schemes.

\section{References}

Agarwal, R. L. and Pawar, A., 1990, Identification of soybean varieties based on seed and seedling characteristics. Seed Res., 18(1): 77-81.

Bacchetta, G., Fenu, G., Grub, O., Mattana, E. and Venora. G., 2011, Species identification by seeds image analysis of Astragalus sect. Melanocercis Bunge (Fabaceae) in Sardinia. Annales Botanici Fennici. in press.

Chakrabarty, S. K. and Agrawal, R. L., 1990, Identification of blackgram varieties utilization of morphological characteristics of seedlings. Seed Res., 17(2):139- 142.

Chavan, N. G., 2010, Characterization of soybean [Glycine $\max$ (L.) Merrill.] varieties through morphological, chemical, molecular markers and image analyzer. M.Sc. (Agri) Thesis, Univ. Agric. Sci., Dharwad, Karnataka. 
Firathgil, E., Sarka, B., Bubnik, Z., Schejbal, M. and Kadlec, P., (2010), Size properties of legume seeds of different varieties using image analysis. J. Food Engg., 99: 445-451.

Grillo, O., Miceli, C. and Venora, G., 2011, Computerised image analysis applied to inspection of vetch seeds for varietal identification. Seed Sci. Technol., 39(2): 490-500.

Mattana, E., Gritto, O., Venom, G. and Bacchetta, G., 2008. Germplasm image analysis of flax. Crop Sci., 65:149-155.

Medina, W., Skurtys, O. and Aguilera, L.M., 2010, Study on image analysis application for identification Quinoa seeds (Chenopodium quinoa) geographical provenance. Food Sci. Technol., 43: 238-246.
Nethra, N., Rajendra, P., Vishwanath, K., Dhanraj, K. N. and Ramegowda, 2007, Identification of rice hybrids and their parental lines based on seed, seedling characters, chemical tests and gel electrophoresis of total soluble seed proteins. Seed Sci. Technol., 35: 176186.

Pascual, V., Oritz, M. J. and Coorreal, E., 1993, Morphometric characterization of seeds of Euphorbia lagascae. Seed Sci. Technol., 21: 53-60.

Rimpi, B., Sharmia, D. D. and Abrata, S. 2008, Characterization of local rice varieties of Assam using morphological markers, Seed Res. 36(2): 218-227.

Zhang, Z. H., Yu, S. B. and Yu, T., 2008, Mapping QTLs for seedling vigour using RILs of rice (Oryza sativa L.). Field Crops Res, 91(3): 161-170.

\section{How to cite this article:}

Elizabeth, N.M. Shakuntala, Basave Gowda, K. Mahantashivayogayya, J.R. Dewan and Sangeeta, I.M. 2018. Evaluation of Rice Genotypes for Seed Morphometric Characters by Using Seed Image Analyser. Int.J.Curr.Microbiol.App.Sci. 7(12): 3368-3375.

doi: https://doi.org/10.20546/ijcmas.2018.712.387 